\title{
On Some Inequalities Involving Three or More Means
}

\author{
Mustapha Raïssouli ${ }^{1,2}$ and Mohamed Chergui ${ }^{3}$ \\ ${ }^{1}$ Department of Mathematics, Science Faculty, Taibah University, P.O. Box 30097, Al Madinah Al Munawwarah 41477, Saudi Arabia \\ ${ }^{2}$ Department of Mathematics, Science Faculty, Moulay Ismail University, 50060 Meknes, Morocco \\ ${ }^{3}$ Department of Mathematics, Centre Régional des Métiers de l'Education et de la Formation, (CRMEF), 14000 Kenitra, Morocco
}

Correspondence should be addressed to Mustapha Raïssouli; raissouli.mustapha@gmail.com

Received 15 April 2016; Accepted 10 July 2016

Academic Editor: Alberto Fiorenza

Copyright (C) 2016 M. Raïssouli and M. Chergui. This is an open access article distributed under the Creative Commons Attribution License, which permits unrestricted use, distribution, and reproduction in any medium, provided the original work is properly cited.

We investigate some results about mean-inequalities involving a large number of bivariate means. As application, we derive a lot of inequalities between four or more means among the standard means known in the literature.

\section{Introduction}

By (bivariate) mean we understand a map $m$ between positive real numbers satisfying the following double inequality:

$$
\min (a, b) \leq m(a, b) \leq \max (a, b), \quad \forall a, b>0 .
$$

As usual, we define continuous (resp., symmetric/ homogeneous) means in the habitual way. The standard examples of such means are given in the following:

$$
\begin{aligned}
A(a, b) & =\frac{a+b}{2} \\
G(a, b) & =\sqrt{a b} \\
H(a, b) & =\frac{2 a b}{a+b} \\
C(a, b) & =\frac{a^{2}+b^{2}}{a+b}, \\
L(a, b) & =\frac{b-a}{\log b-\log a} \\
P(a, b) & =\frac{b-a}{4 \arctan \sqrt{b / a}-\pi} \\
& =\frac{b-a}{2 \arcsin ((b-a) /(b+a))}
\end{aligned}
$$

$$
\begin{aligned}
T(a, b) & =\frac{b-a}{2 \arctan (b / a)-\pi / 2} \\
& =\frac{b-a}{2 \arctan ((b-a) /(b+a))}, \\
M(a, b) & =\frac{b-a}{2 \sinh ^{-1}((b-a) /(b+a))},
\end{aligned}
$$

with $L(a, a)=P(a, a)=T(a, a)=M(a, a)=a$, and they are known as the arithmetic mean, geometric mean, harmonic mean, contraharmonic mean, logarithmic mean, first Seiffert mean [1], second Seiffert mean [2], and Neuman-Sándor mean [3], respectively. Other examples of means (not needed here) can be found in the literature; see [4], for instance, and the references cited therein.

The two means,

$$
\begin{aligned}
& U(a, b)=\frac{b-a}{\sqrt{2} \arctan ((b-a) / \sqrt{2 a b})}, \\
& U(a, a)=a, \\
& V(a, b)=\frac{b-a}{\sqrt{2} \sinh ^{-1}((b-a) / \sqrt{2 a b})}, \\
& V(a, a)=a,
\end{aligned}
$$


were introduced in [5] (see page 9 and page 24, resp.). These two means are included in the so-called Seiffert type means discussed in [6].

As usual, we identify a mean $m$ with its value at $(a, b)$ by setting $m:=m(a, b)$ for the sake of simplicity. If $m_{1}$ and $m_{2}$ are two means, we write $m_{1}<m_{2}$ for meaning that $m_{1}(a, b)<$ $m_{2}(a, b)$ for all $a, b>0$ with $a \neq b$. Two different means $m_{1}$ and $m_{2}$ are called comparable if $m_{1}<m_{2}$ or $m_{2}<m_{1}$ holds. As it is well-known, means $A, G, H, L, P, M$, and $T$ are mutually comparable with $H<G<L<P<A<M<T$. Concerning means $U$ and $V$ we have $L<V<P<U<$ $M$. However, the means $U$ and $A$ are not comparable; see Section 5.

The notation $m^{*}$ refers to the dual mean of $m$ defined by $m^{*}(a, b)=\left(m\left(a^{-1}, b^{-1}\right)\right)^{-1}$ for all $a, b>0$. As it is well-known, if $m$ is symmetric and homogeneous then so is $m^{*}$, with $m^{*}(a, b)=a b / m(a, b)$ which we briefly write as $m^{*}=G^{2} / m$.

For over the last years, mean-theory has been the subject of intensive research. It has been proved, throughout a large number of works, that mean-theory is useful from theoretical point of view and for practical purposes. The most interesting subject in mean-theory is to investigate mean-inequalities that occur in a primordial place in the literature. For instance, many inequalities involving trigonometric/hyperbolic functions can be derived from mean-inequalities in a simple and fast way. As far as we know, published results about comparison of the previous standard means concern meaninequalities involving only two or three means. Inspired by an approach, recently introduced by the first author in [7], we present here some results about inequalities involving a large number of means. In particular, inequalities involving four or more means among the standard means $A, G, L, P, T$, and $M$ are discussed. Inequalities involving the means $U$ and $V$ are also obtained.

\section{Background Material}

In this section, we state some results that will be needed throughout the following. We begin by recalling the following result; see [7].

Theorem 1. Let $m$ be a continuous homogeneous symmetric mean. Then the binary map $m^{\sigma}$ defined by $m^{\sigma}(a, a)=a$ and

$$
\left(m^{\sigma}(a, b)\right)^{-1}=\frac{1}{b-a} \int_{1}^{b / a} m\left(1, \frac{1}{t^{2}}\right) d t
$$

for all $a, b>0$ with $a \neq b$ is a continuous homogeneous symmetric mean (called the integral mean-transform of $m$ ).

As example, we have $A^{\sigma}=H, G^{\sigma}=L$, and $H^{\sigma}=T$. The mean-map $m \mapsto m^{\sigma}$ is pointwisely strictly decreasing; that is, if $m_{1}$ and $m_{2}$ are two continuous homogeneous symmetric means such that $m_{1}<m_{2}$, then we have $m_{1}^{\sigma}>m_{2}^{\sigma}$.

As pointed out in [7], a continuous homogeneous symmetric mean will be called regular mean, for the sake of simplicity. A regular mean $m$ will be called $\sigma$-regular if the map $x \mapsto m(x, 1)$ is continuously differentiable on $(0, \infty)$ and the function $f_{m}$ (called the generated function of $m$ ) defined by

$$
f_{m}(x)=\frac{d}{d x}\left(\frac{x-1}{m(x, 1)}\right)
$$

for all $x>0$, with $f_{m}(1)=1$, satisfies

$$
\min \left(1, \frac{1}{x^{2}}\right) \leq f_{m}(x) \leq \max \left(1, \frac{1}{x^{2}}\right)
$$

for all $x>0$. The previous means are regular and $\sigma$-regular, except $C$ which is not $\sigma$-regular (see [7, Examples 3.1, 3.2, 3.3]).

The following result has also been proved in [7].

Theorem 2. Let $m$ be a $\sigma$-regular mean with its generated function $f_{m}$. Then the binary map $r_{m}$ defined by

$$
r_{m}(a, b)=b f_{m}\left(\sqrt{\frac{b}{a}}\right)
$$

for all $a, b>0$ is a regular mean with $r_{m}^{\sigma}=m$.

If we denote by $\mathscr{M}_{r}$ and $\mathscr{M}_{\sigma}$ the sets of all regular means and $\sigma$-regular means, respectively, then the mean-map $m \mapsto$ $m^{\sigma}$ is a bijection from $\mathscr{M}_{r}$ into $\mathscr{M}_{\sigma}$ and we can write (see [7])

$$
\begin{gathered}
\left(r_{m}^{\sigma}=m, r_{m} \in \mathscr{M}_{r}\right) \Longleftrightarrow \\
\left(r_{m}=m^{-\sigma}, m \in \mathscr{M}_{\sigma}\right) .
\end{gathered}
$$

With this, the following result, which will be needed throughout the following, has been established in [7].

Theorem 3. The following relationships hold:

$$
\begin{aligned}
& H^{-\sigma}=A, \\
& L^{-\sigma}=G \\
& T^{-\sigma}=H \\
& A^{-\sigma}=\left(\frac{A+G}{2}\right)^{*}=\frac{2 G^{2}}{A+G}, \\
& G^{-\sigma}=\left(\frac{A G+G^{2}}{2}\right)^{1 / 2}, \\
& P^{-\sigma}=\left(\left(\frac{A+G}{2}\right)^{*} G\right)^{1 / 2}=\left(\frac{2 G^{3}}{A+G}\right)^{1 / 2}, \\
& M^{-\sigma}=\left(\left(\frac{A^{2}+A G}{2}\right)^{1 / 2}\right)^{*}=\left(\frac{2 G^{4}}{A^{2}+A G}\right)^{1 / 2},
\end{aligned}
$$

where the notation $m^{*}$ refers to the dual mean of $m$.

If, for $m \in \mathscr{M}_{\sigma}$, we set

$$
F_{m}(z):=\frac{m^{-\sigma}}{G}, \quad \text { with } z=\frac{A}{G} \geq 1
$$


it is easy to see that if $m_{1}, m_{2} \in \mathscr{M}_{\sigma}$ then we have [7]

$$
\left(F_{m_{1}}(z)>F_{m_{2}}(z), \forall z>1\right) \Longrightarrow m_{1}<m_{2} .
$$

Finally, we recall the following result; see, for example, [7].

Theorem 4. The following relationships are met:

$$
\begin{aligned}
& F_{L}(z)=1, \\
& F_{H}(z)=z, \\
& F_{T}(z)=\frac{1}{z} \\
& F_{A}(z)=\frac{2}{z+1}, \\
& F_{G}(z)=\sqrt{\frac{z+1}{2}}, \\
& F_{P}(z)=\sqrt{\frac{2}{z+1}}, \\
& F_{M}(z)=\sqrt{\frac{2}{z^{2}+z}}, \\
& F_{U}(z)=\frac{1}{z} \sqrt{\frac{z+1}{2}}, \\
& F_{V}(z)=\sqrt{\frac{z+1}{2 z}} .
\end{aligned}
$$

Using the previous result, it is easy to see that, for all $z>1$, we have

$$
\begin{aligned}
F_{T}(z) & <F_{M}(z)<F_{A}(z)<F_{P}(z)<F_{L}(z)<F_{G}(z) \\
& <F_{H}(z), \\
F_{M}(z) & <F_{U}(z)<F_{P}(z)<F_{V}(z)<F_{L}(z),
\end{aligned}
$$

which, with (11), immediately implies (simultaneously and in a fast way) the known chains $H<G<L<P<A<M<$ $T$ and $L<V<P<U<M$, respectively. The previous inequalities are graphically illustrated in Figure 1.

\section{Some Needed Results}

In this section we present some results that will be needed for obtaining a lot of mean-inequalities involving four or more means among the previously mentioned means. We preserve the same notations as in the previous sections. We begin by stating the following result.

Theorem 5. Let $m_{1}, m_{2}, \ldots, m_{n}$ be $n$ regular means and $\lambda_{1}, \lambda_{2}, \ldots, \lambda_{n} \in(0,1)$ such that $\sum_{i=1}^{n} \lambda_{i}=1$. Then one has

$$
\left(\sum_{i=1}^{n} \lambda_{i} m_{i}\right)^{\sigma} \leq \sum_{i=1}^{n} \lambda_{i}\left(m_{i}\right)^{\sigma}
$$

If moreover two different means among $m_{1}, m_{2}, \ldots, m_{n}$ are comparable then (14) is strict.

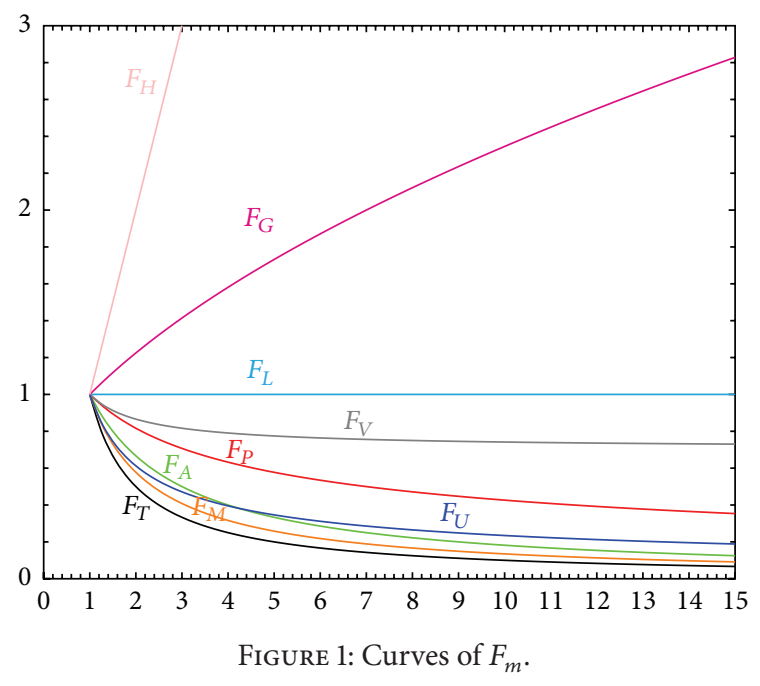

Proof. First, it is easy to see that $\sum_{i=1}^{n} \lambda_{i} m_{i}$ is a regular mean. By definition, for all $a, b>0, a \neq b$, we have

$$
\begin{aligned}
& \left(\left(\sum_{i=1}^{n} \lambda_{i} m_{i}\right)^{\sigma}(a, b)\right)^{-1} \\
& =\frac{1}{b-a} \int_{1}^{b / a} \sum_{i=1}^{n} \lambda_{i} m_{i}\left(1, \frac{1}{t^{2}}\right) d t .
\end{aligned}
$$

This, with the linearity of integral, gives

$$
\left(\left(\sum_{i=1}^{n} \lambda_{i} m_{i}\right)^{\sigma}(a, b)\right)^{-1}=\sum_{i=1}^{n} \lambda_{i}\left(m_{i}^{\sigma}(a, b)\right)^{-1} .
$$

Taking inverse-sides of (16) and using the fact that the realvalued function $x \mapsto 1 / x$ is (strictly) convex on $(0, \infty)$, generalized Jensen's inequality asserts that

$$
\left(\sum_{i=1}^{n} \lambda_{i} m_{i}\right)^{\sigma}(a, b) \leq \sum_{i=1}^{n} \lambda_{i} m_{i}^{\sigma}(a, b) .
$$

Now, if two means among $m_{1}, m_{2}, \ldots, m_{n}$ are different and comparable, say $m_{i}(a, b)<m_{j}(a, b)$ for all $a, b>0$ with $a \neq b$ and some $i \neq j$, then the strict convexity of $x \mapsto 1 / x$ implies that previous Jensen's inequality is strict and the proof is complete.

For practical purposes, the following corollary is of interest.

Corollary 6. Let $m_{1}, m_{2}, \ldots, m_{n}$ and $m$ be $\sigma$-regular means. Assume that, for some $\lambda_{1}, \lambda_{2}, \ldots, \lambda_{n} \in(0,1)$ such that $\sum_{i=1}^{n} \lambda_{i}=1$, the inequality

$$
\sum_{i=1}^{n} \lambda_{i} F_{m_{i}}(z)<F_{m}(z)
$$

holds true for all $z>1$. Then we have

$$
m<\sum_{i=1}^{n} \lambda_{i} m_{i}
$$


Proof. Since $F_{m}(z):=m^{-\sigma} / G$, with $z=A / G$, then (18) can be written as follows:

$$
\sum_{i=1}^{n} \lambda_{i} m_{i}^{-\sigma}<m^{-\sigma}
$$

This, with (14) and the fact that the mean-map $m \mapsto m^{\sigma}$ is pointwisely strictly decreasing, yields

$$
\begin{aligned}
m & =\left(m^{-\sigma}\right)^{\sigma}<\left(\sum_{i=1}^{n} \lambda_{i} m_{i}^{-\sigma}\right)^{\sigma} \leq \sum_{i=1}^{n} \lambda_{i}\left(m_{i}^{-\sigma}\right)^{\sigma} \\
& =\sum_{i=1}^{n} \lambda_{i} m_{i},
\end{aligned}
$$

which implies the desired result.

The following example shows that the reverse statement of the previous corollary is not always true.

Example 7. It is easy to see that the inequality

$$
\frac{2}{3} x+\frac{2}{3}\left(\frac{1}{2 x}\right)^{1 / 2} \geq 1
$$

holds for all $x>0$ with equality if and only if $x=1 / 2$. This, with the fact that $G /(A+G)(a, b) \neq 1 / 2$ for $a \neq b$, yields

$$
\frac{1}{3}\left(\frac{2 G}{A+G}\right)+\frac{2}{3}\left(\frac{A+G}{2 G}\right)^{1 / 2}>1 .
$$

Writing this inequality in the form

$$
\frac{1}{3}\left(\frac{2 G^{2}}{A+G}\right)+\frac{2}{3}\left(\frac{A G+G^{2}}{2}\right)^{1 / 2}>G,
$$

we then obtain (by virtue of Theorem 3)

$$
\frac{1}{3} A^{-\sigma}+\frac{2}{3} G^{-\sigma}>L^{-\sigma}
$$

This, with the known inequality $L<(1 / 3) A+(2 / 3) G$ (see, e.g., $[8,9])$, justifies our claim.

Theorem 8. Let $m_{1}, m_{2}, \ldots, m_{n}$ be $n$ regular means and $\lambda_{1}, \lambda_{2}, \ldots, \lambda_{n} \in(0,1)$ such that $\sum_{i=1}^{n} \lambda_{i}=1$. Then there holds

$$
\left(\prod_{i=1}^{n} m_{i}^{\lambda_{i}}\right)^{\sigma} \geq \prod_{i=1}^{n}\left(m_{i}^{\sigma}\right)^{\lambda_{i}} \text {. }
$$

If moreover two means among $m_{1}, m_{2}, \ldots, m_{n}$ are different then (26) is strict.

Proof. Clearly, $\prod_{i=1}^{n} m_{i}^{\lambda_{i}}$ is a regular mean. Without loss of generality, we can assume that $a<b$. By definition of the integral transform-mean we have

$$
\begin{aligned}
& \left(\left(\prod_{i=1}^{n} m_{i}^{\lambda_{i}}\right)^{\sigma}(a, b)\right)^{-1} \\
& \quad=\frac{1}{b-a} \int_{1}^{b / a} \prod_{i=1}^{n} m_{i}^{\lambda_{i}}\left(1, \frac{1}{t^{2}}\right) d t .
\end{aligned}
$$

According to generalized Hölder's inequality for integrals, the latter inequality yields

$$
\begin{aligned}
& \left(\left(\prod_{i=1}^{n} m_{i}^{\lambda_{i}}\right)^{\sigma}(a, b)\right)^{-1} \\
& \quad \leq \frac{1}{b-a} \prod_{i=1}^{n}\left(\int_{1}^{b / a}\left(m_{i}^{\lambda_{i}}\left(1, \frac{1}{t^{2}}\right)\right)^{1 / \lambda_{i}} d t\right)^{\lambda_{i}} .
\end{aligned}
$$

Since $\sum_{i=1}^{n} \lambda_{i}=1$ the last inequality becomes

$$
\begin{aligned}
& \left(\left(\prod_{i=1}^{n} m_{i}^{\lambda_{i}}\right)^{\sigma}(a, b)\right)^{-1} \\
& \leq \prod_{i=1}^{n}\left(\frac{1}{b-a} \int_{1}^{b / a} m_{i}\left(1, \frac{1}{t^{2}}\right) d t\right)^{\lambda_{i}},
\end{aligned}
$$

or again

$$
\left(\left(\prod_{i=1}^{n} m_{i}^{\lambda_{i}}\right)^{\sigma}(a, b)\right)^{-1} \leq \prod_{i=1}^{n}\left(\left(m_{i}^{\sigma}(a, b)\right)^{-1}\right)^{\lambda_{i}} .
$$

Taking inverses of the two sides of this latter inequality we find

$$
\begin{aligned}
\left(\prod_{i=1}^{n} m_{i}^{\lambda_{i}}\right)^{\sigma}(a, b) & \geq \prod_{i=1}^{n}\left(m_{i}^{\sigma}(a, b)\right)^{\lambda_{i}} \\
& :=\prod_{i=1}^{n}\left(m_{i}^{\sigma}\right)^{\lambda_{i}}(a, b) .
\end{aligned}
$$

Now, if the means $m_{1}, m_{2}, \ldots, m_{n}$ are not all equal then we can see that Hölder's inequality is here strict, thus completing the proof.

The following corollary is of interest for practical purposes.

Corollary 9. Let $m_{1}, m_{2}, \ldots, m_{n}$ and $m$ be $\sigma$-regular means. Assume that, for some $\lambda_{1}, \lambda_{2}, \ldots, \lambda_{n} \in(0,1)$ such that $\sum_{i=1}^{n} \lambda_{i}=1$, the inequality

$$
F_{m}(z) \leq \prod_{i=1}^{n}\left(F_{m_{i}}(z)\right)^{\lambda_{i}}
$$

holds true for all $z \geq 1$. Then we have

$$
\prod_{i=1}^{n} m_{i}^{\lambda_{i}} \leq m
$$

If moreover there exist two means among $m_{1}, m_{2}, \ldots, m_{n}$ which are different then (33) is strict.

Proof. In similar way as the proof of Corollary 6, we deduce the desired result from the previous theorem. Detail is simple and therefore omitted here.

We end this section by stating the following remark.

Remark 10. It is worth mentioning that the mean-inequality (33) is strict even if (32) is an equality, provided two means among $m_{1}, m_{2}, \ldots, m_{n}$ are different. 


\section{Inequalities Involving $G, A, L, P, M$, and $T$}

This section is devoted to investigating some applications for mean-inequalities. As already pointed before, we give meaninequalities involving some means among those recalled in the previous introduction. Concerning mean-inequalities in a geometric combination involving only three means,

$$
\begin{gathered}
G P<L^{2}, \\
P M<A^{2}, \\
A T<M^{2}, \\
L T<A^{2}, \\
L A<P^{2}, \\
A^{2} G<P^{3}
\end{gathered}
$$

have been proved in [10, 11]; see also [7]. Other inequalities in geometric combination, such as $\left(Q A^{2}\right)^{1 / 3}<M<(Q A)^{1 / 2}$, can be found in the literature. Here $Q$ refers to the quadratic mean; that is, $Q:=Q(a, b)=\sqrt{\left(a^{2}+b^{2}\right) / 2}$. In a parallel way, mean-inequalities in a (convex) combination sum were the subject of many published papers. See [12] and the related references cited therein.

The following result gives more mean-inequalities involving only three means among $G, A, L, P, M$, and $T$.

Theorem 11. The following inequalities hold true:

$$
\begin{gathered}
L^{2} M<P^{3}, \\
L^{3} T<P^{4}, \\
G^{3} M<L^{4}, \\
G^{4} T<L^{5}, \\
P T^{2}<M^{3}, \\
G T^{4}<M^{5}, \\
P^{2} T<A^{3} .
\end{gathered}
$$

Proof. Let us prove the inequality $L^{2} M<P^{3}$. Using Theorem 4 , simple observation leads to the fact that

$$
\begin{aligned}
\left(F_{L}(z)\right)^{2} F_{M}(z) & =F_{M}(z)=\sqrt{\frac{2}{z^{2}+z}}=\frac{1}{\sqrt{z}} \sqrt{\frac{2}{z+1}} \\
& \geq \frac{2}{z+1} \sqrt{\frac{2}{z+1}}=\left(F_{P}(z)\right)^{3}
\end{aligned}
$$

hold for all $z \geq 1$. This, with Corollary 9, yields the first meaninequality. The other mean-inequalities can be proved in the same way. Detail is simple and therefore omitted here with the aim of not lengthening this paper.
Remark 12. In [13] the authors showed that $P^{\alpha} T^{1-\alpha}<M$ if and only if $\alpha \geq 1 / 3$. This means that the mean-inequality $P T^{2}<M^{3}$ (previously mentioned) is the best possible.

Otherwise, it is worth mentioning that certain inequalities of the above theorem can be deduced from those of (34). As example, from $P M<A^{2}$, that is, $M<A^{2} / P$, one has $L^{2} M<L^{2} A^{2} / P<P^{3}$, since this latter inequality is equivalent to $L A<P^{2}$. However, our aim here is to show how to apply the same procedure, described by the present approach, for proving old/new mean-inequalities in a simple and fast way.

Similarly, we can find a lot of mean-inequalities involving four means among the standard means $G, A, L, P, M$, and $T$. For instance, we cite the following.

Theorem 13. The following mean-inequalities are satisfied:

$$
\begin{gathered}
P^{2} A T^{2}<M^{5}, \\
G^{6} A T<L^{8}, \\
G^{2} L T<P^{4}, \\
L^{2} M^{2} T<A^{5}, \\
G^{4} M^{2}<L^{5} A .
\end{gathered}
$$

Proof. We show $P^{2} A T^{2}<M^{5}$ and $G^{4} M^{2}<L^{5} A$, for example. The proofs of the other inequalities are analogue.

For all $z \geq 1$, we can easily verify that (by Theorem 4 )

$$
\left(F_{P}(z)\right)^{2} F_{A}(z)\left(F_{T}(z)\right)^{2}=\left(F_{M}(z)\right)^{4} \geq\left(F_{M}(z)\right)^{5} .
$$

By Corollary 9 we immediately obtain the first inequality. For the second one, we have

$$
\left(F_{G}(z)\right)^{2} F_{H}(z)\left(F_{M}(z)\right)^{2}=1=\left(F_{L}(z)\right)^{5},
$$

which by the same reason as previous implies $G^{2} H M^{2}<L^{5}$. Now, getting $H=G^{2} / A$ in this latter inequality we then obtain the second desired inequality.

We left to the reader the routine task for obtaining more inequalities in an analogous way. Similarly, inequalities involving five means can be obtained as well. The following result lists some of them.

Theorem 14. The following mean-inequalities hold:

$$
\begin{aligned}
G^{2} M T & <P^{3} A, \\
G^{10} P^{2} A M^{2} & <L^{15}, \\
G^{12} P^{2} M^{2} T & <L^{17}, \\
G^{16} A M^{2} T^{2} & <L^{21}, \\
G^{2} L^{3} M^{2} T & <P^{8}, \\
L P^{2} M^{2} T & <A^{6}, \\
G^{3} P T & <L^{4} A .
\end{aligned}
$$


Proof. We show $G^{2} M T<P^{3} A$ and $L P^{2} M^{2} T<A^{6}$, for instance. For all $z \geq 1$, we can easily see that (always by Theorem 4)

$$
F_{M}(z) F_{T}(z) F_{H}(z)=F_{M}(z) \geq\left(F_{P}(z)\right)^{3},
$$

which, with Corollary 9, yields $M T H<P^{3}$. Getting $H=$ $G^{2} / A$, we deduce the first desired inequality. The second one can be similarly obtained from the following:

$$
F_{L}(z)\left(F_{P}(z)\right)^{2}\left(F_{M}(z)\right)^{2} F_{T}(z) \geq\left(F_{A}(z)\right)^{6} .
$$

Concerning mean-inequalities involving all the six means $A, G, L, P, M$, and $T$, we have the following result.

Theorem 15. The three following mean-inequalities hold:

$$
\begin{gathered}
G^{2} L^{2} M^{2} T<P^{6} A, \\
G^{14} P^{2} A M^{2} T<L^{20}, \\
G^{10} P^{2} M^{2} T<L^{14} A .
\end{gathered}
$$

Proof. We just show the first inequality, for example. In similar way as previous, we have (for all $z \geq 1$ )

$$
\begin{aligned}
& \left(F_{M}(z)\right)^{2}\left(F_{L}(z)\right)^{2} F_{T}(z) F_{H}(z)=\left(F_{M}(z)\right)^{2} \\
& \quad \geq\left(F_{P}(z)\right)^{6},
\end{aligned}
$$

from which we get $M^{2} L^{2} T H<P^{6}$. Replacing $H$ by $G^{2} / A$ we obtain the desired inequality.

We end this section by stating the following interesting result.

Theorem 16. For every real number $q \geq 1$ we have

$$
G A^{q+1} L^{q-1}<P^{2 q+1}
$$

Proof. By Theorem 4, simple computation leads to

$$
F_{G}(z)\left(F_{A}(z)\right)^{q+1}\left(F_{L}(z)\right)^{q-1}=\left(F_{P}(z)\right)^{2 q+1}
$$

for all $z \geq 1$. This, with Corollary 9, yields the desired meaninequality.

Remark 17. Inequality (45) contains some known meaninequalities shown in the literature by different ways. In fact, taking $q=1$ it yields $G A^{2}<P^{3}$ which is the last inequality in (34). Writing it in the equivalent form

$$
G^{1 / q} A^{1+1 / q} L^{1-1 / q}<P^{2+1 / q}
$$

and then letting $q \rightarrow \infty$ we find again $A L<P^{2}$; see (34). For $q=2$, it gives $G A^{3} L<P^{5}$.

\section{Inequalities Involving $U$ and $V$}

As already pointed before, the means $U$ and $V$ satisfy the following chain of inequalities:

$$
(G<) L<V<P<U<M(<T) .
$$

We notice that means $A$ and $U$ are not comparable. In fact, in [14] the authors proved that the double inequality

$$
M_{p}(a, b)<U(a, b)<M_{q}(a, b)
$$

takes place if and only if $p \leq 2 \log 2 /(2 \log \pi-\log 2)=$ $0.8684 \ldots$ and $q \geq 4 / 3$, where $M_{p}(a, b)=\left[\left(a^{p}+\right.\right.$ $\left.\left.b^{p}\right) / 2\right]^{1 / p}, p \neq 0$, and $M_{0}(a, b)=\sqrt{a b}$, is the $p$ thpower mean. Therefore, $U$ and $A$ are not comparable because $A(a, b):=(a+b) / 2=M_{1}(a, b)$.

For recent developments about means $U$ and $V$ we refer the reader to [14-18]. As far as we know, mean-inequalities involving three or more means and including $U$ and/or $V$ are not investigated in the literature yet. Adopting the previous approach, we can give a lot of mean-inequalities involving $U$ and $V$. We start with the case of convex arithmetic combination.

Theorem 18. The following inequalities hold:

$$
\begin{aligned}
& V<\frac{L+U}{2}, \\
& U<\frac{A+T}{2} .
\end{aligned}
$$

Proof. For all $z>1$, we have (by Theorem 4)

$$
F_{V}(z)=\sqrt{\frac{z+1}{2 z}}=\sqrt{\frac{1}{2}+\frac{1}{2} \frac{1}{z}}>\frac{1}{2}+\frac{1}{2} \frac{1}{\sqrt{z}},
$$

since the real-function $x \mapsto \sqrt{x}$ is strictly concave on $(0, \infty)$. A simple verification leads to

$$
\frac{1}{\sqrt{z}}>\frac{1}{z} \sqrt{\frac{z+1}{2}}=F_{U}(z)
$$

for all $z>1$. This, with $F_{L}(z)=1$, gives

$$
F_{V}(z)>\frac{1}{2} F_{L}(z)+\frac{1}{2} F_{U}(z)
$$

which, with Corollary 6, yields the first inequality of (50). The second inequality of (50) can be proved in a similar way.

Now, we give some inequalities in convex geometric combination between three means including $U$ and $V$. 
Theorem 19. The following mean-inequalities hold:

$$
\begin{gathered}
G U<V^{2}, \\
V M<A^{2}, \\
G V<L^{2}, \\
V M<U^{2}, \\
V^{2} T<A^{3}, \\
L^{2} U<P^{3}, \\
V^{2} T<U^{3}, \\
G^{2} T<V^{3} .
\end{gathered}
$$

Proof. We will show the inequalities $G U<V^{2}$ and $V M<U^{2}$, for instance. For all $z \geq 1$, it is easy to see that

$$
\begin{aligned}
& F_{G}(z) F_{U}(z)=\left(F_{V}(z)\right)^{2}, \\
& F_{V}(z) F_{M}(z) \geq\left(F_{U}(z)\right)^{2} .
\end{aligned}
$$

These, with Corollary 9 , yield the desired inequalities.

Inequalities involving four or more means including $U$ and $V$ can be obtained in a similar way as previous. As examples, we cite the following:

$$
\begin{gathered}
G V^{2} P^{3}<L^{6}, \\
G L U<V^{3}, \\
G^{2} L A<U^{4}, \\
G^{5} U M<V^{7}, \\
V^{6} P^{2} T<U^{9}, \\
V^{2} P^{6} T<M^{9}, \\
V^{2} U^{2} T<A^{5}, \\
V^{2} P M<A^{4}, \\
G L V U A<P^{5}, \\
G^{6} V^{2} M^{2} T<L^{8} A^{3} .
\end{gathered}
$$

Other more interesting examples are given in the following result.

Theorem 20. The two inequalities

$$
\begin{aligned}
G^{2 q} U^{2} T^{q-1} & <V^{3 q+1}, \\
G^{4 q} A M^{2 q-2} T^{q+1} & <U^{7 q}
\end{aligned}
$$

hold for each $q \geq 1$, while

$$
G^{2 q+2} U^{2 q} T<V^{3 q+2} A^{q+1}
$$

is valid for every $q \geq 0$.
Proof. According to Theorem 4 we have (for all $z \geq 1$ )

$$
\begin{aligned}
& \left(F_{G}(z)\right)^{2 q}\left(F_{U}(z)\right)^{2}\left(F_{T}(z)\right)^{q-1}=\left(\frac{z+1}{2 z}\right)^{q+1} \\
& =\left(F_{V}(z)\right)^{2 q+2} \geq\left(F_{V}(z)\right)^{3 q+1},
\end{aligned}
$$

since $3 q+1 \geq 2 q+2$ and $F_{V}(z) \leq 1$ for all $z \geq 1$. This, with Corollary 9, gives (57). Inequalities (58) and (59) can be proved in a similar way.

For particular cases of the parameter $q$, we can find again some mean-inequalities among those stated in the previous results. The following example explains this latter situation.

Example 21. (1) With $q=1$, (57) gives $G U<V^{2}$ which is the first inequality of (54). Writing it in the form

$$
T^{1-1 / q} G^{2} U^{2 / q}<V^{3+1 / q}
$$

and getting $q \rightarrow \infty$ we find $G^{2} T<V^{3}$ which is the last inequality in (54).

(2) With $q=0$, (59) yields $G^{2} T<V^{2} A$ while with $q=1$ it gives $G^{4} U^{2} T<V^{5} A^{2}$. Writing (59) in the equivalent form

$$
G^{2+2 / q} U^{2} T^{1 / q}<V^{3+2 / q} A^{1+1 / q}
$$

and then letting $q \rightarrow \infty$ we obtain $G^{2} U^{2}<V^{3} A$.

Other mean-inequalities can be deduced from (58) in a similar way. We left it to the reader.

It is clear that whenever we have a mean-inequality involving $n+1$ means we can deduce from it a meaninequality involving only $p \leq n$ means, provided the involved $n+1$ means are mutually comparable. The following example explains more precisely such situation.

Example 22. Let us consider the first inequality of Theorem 15; that is, $G^{2} L^{2} M^{2} T<P^{6} A$. This, with $A<T$, implies that $G L M<P^{3}$.

As it is well-known, mean-inequalities could be good tool for proving some inequalities involving trigonomet$\mathrm{ric} /$ hyperbolic functions whose proofs in a direct way present serious difficulties. Let us observe this situation in the following example.

Example 23. Let us consider (45); that is,

$$
G A^{q+1} L^{q-1}<P^{2 q+1}, \quad \forall q \geq 1 .
$$

(i) Let $x \in(0, \pi / 2)$ and set $a=1+\sin x, b=1-\sin x$. Simple computation leads to

$$
\begin{aligned}
& A=1, \\
& G=\cos x, \\
& P=\frac{\sin x}{x}, \\
& L=\frac{\sin x}{\operatorname{arctanh}(\sin x)} .
\end{aligned}
$$


Substituting these in the previous mean-inequality we obtain

$$
\begin{aligned}
& x^{2 q+1} \cos x<(\sin x)^{q+2}(\operatorname{arctanh}(\sin x))^{q-1}, \\
& \forall q \geq 1, \forall x \in\left(0, \frac{\pi}{2}\right) .
\end{aligned}
$$

(ii) Let $x \neq 0$ and $a=e^{x}, b=e^{-x}$. We then have

$$
\begin{aligned}
& G=1, \\
& A=\cosh x, \\
& L=\frac{\sinh x}{x}, \\
& P=\frac{\sinh x}{\arcsin (\tanh x)},
\end{aligned}
$$

which when substituted in the previous mean-inequality yield

$$
\begin{array}{r}
(\cosh x)^{q+1}(\arcsin (\tanh x))^{2 q+1}<x^{q-1}(\sinh x)^{q+2} \\
\forall q \geq 1, \forall x>0 .
\end{array}
$$

Now, take $q=1$. Inequality (65) implies

$$
x^{3} \cos x<\sin ^{3} x, \quad \forall x \in\left(0, \frac{\pi}{2}\right)
$$

while (67) yields

$$
(\cosh x)^{2}(\arcsin (\tan x))^{3}<(\sinh x)^{3}, \quad \forall x>0
$$

which are well-known inequalities. We left to the reader the routine task for deducing trigonometric/hyperbolic inequalities from mean-inequalities (57), (58), and (59).

\section{Competing Interests}

The authors declare that there is no conflict of interests regarding the publication of this paper.

\section{References}

[1] H. J. Seiffert, "Problem 887," Nieuw Archief voor Wiskunde, vol. 11, p. 176, 1993.

[2] H. J. Seiffert, “Aufgabe 16," Die Wurzel, vol. 29, p. 87, 1995.

[3] E. Neuman and J. Sándor, "On the Schwab-Borchardt mean," Mathematica Pannonica, vol. 14, no. 2, pp. 253-266, 2003.

[4] P. S. Bullen, Handbook of Means and Their Inequalities, Mathematics and Its Applications, Springer, 2nd edition, 1987.

[5] Z. H. Yang, "Three families of two parameter means constructed by trigonometric functions," Journal of Inequalities and Applications, vol. 2013, article 541, 27 pages, 2013.

[6] G. Toader, "Seiffert type means," Nieuw Archief voor Wiskunde, vol. 17, no. 3, pp. 379-382, 1999.

[7] M. Raïssouli, "On an approach in service of mean-inequalities," Journal of Mathematical Inequalities, vol. 10, no. 1, pp. 83-99, 2016.
[8] B. C. Carlson, “The Logarithmic mean," The American Mathematical Monthly, vol. 79, pp. 615-618, 1972.

[9] M. Raïssouli, "Refinements for mean-inequalities via the stabilizability concept," Journal of Inequalities and Applications, vol. 2012, article 55, 18 pages, 2012.

[10] E. Neuman and J. Sándor, "On the Schwab-Borchardt mean II," Mathematica Pannonica, vol. 17, no. 1, pp. 49-59, 2006.

[11] J. Sándor, “On certain inequalities for means III," Archiv der Mathematik, vol. 76, no. 1, pp. 34-40, 2001.

[12] E. Neuman, "A note on a certain bivariate mean," Journal of Mathematical Inequalities, vol. 6, no. 4, pp. 637-643, 2012.

[13] H.-Y. Huang, N. Wang, and B.-Y. Long, "Optimal bounds for Neuman-Sándor mean in terms of the geometric convex combination of two Seiffert means," Journal of Inequalities and Applications, vol. 2016, 14 pages, 2016.

[14] Z.-H. Yang, L.-M. Wu, and Y.-M. Chu, "Optimal power mean bounds for Yang mean," Journal of Inequalities and Applications, vol. 2014, p. 401, 2014.

[15] J. F. Li, Z. H. Yang, and Y. M. Chu, "Optimal power mean bounds for the second yang mean," Journal of Inequalities and Applications, vol. 2016, article 31, 9 pages, 2016.

[16] W.-M. Qian, Y.-M. Chu, and X.-H. Zhang, "Sharp oneparameter mean bounds for Yang mean," Mathematical Problems in Engineering, vol. 2016, Article ID 1579468, 5 pages, 2016.

[17] W.-M. Qian and Y.-M. Chu, "Best possible bounds for Yang mean using generalized logarithmic mean," Mathematical Problems in Engineering, vol. 2016, Article ID 8901258, 7 pages, 2016.

[18] S.-S. Zhou, W.-M. Qian, Y.-M. Chu, and X.-H. Zhang, "Sharp power-type Heronian mean bounds for the Sándor and Yang means," Journal of Inequalities and Applications, vol. 2015, article 159, 10 pages, 2015. 


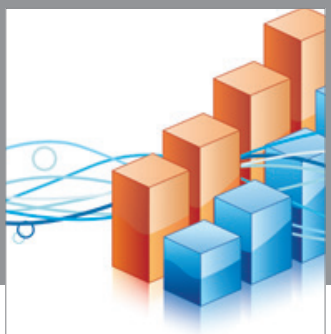

Advances in

Operations Research

vatem alat4

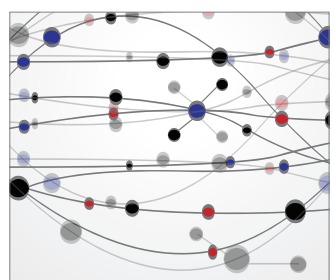

\section{The Scientific} World Journal
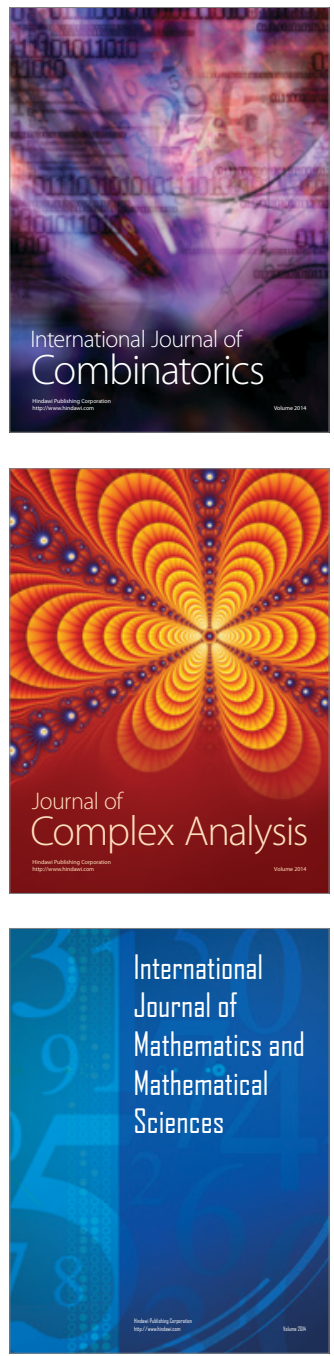
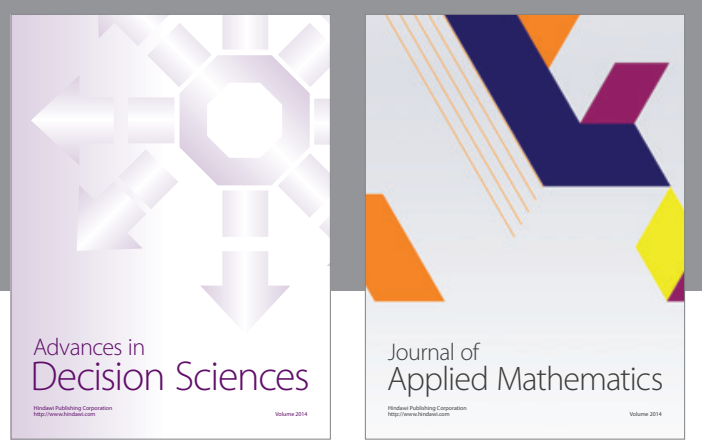

Algebra

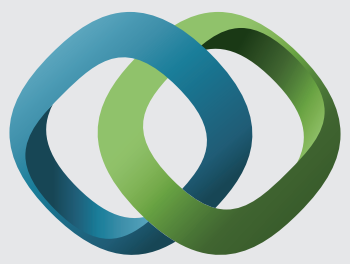

\section{Hindawi}

Submit your manuscripts at

http://www.hindawi.com
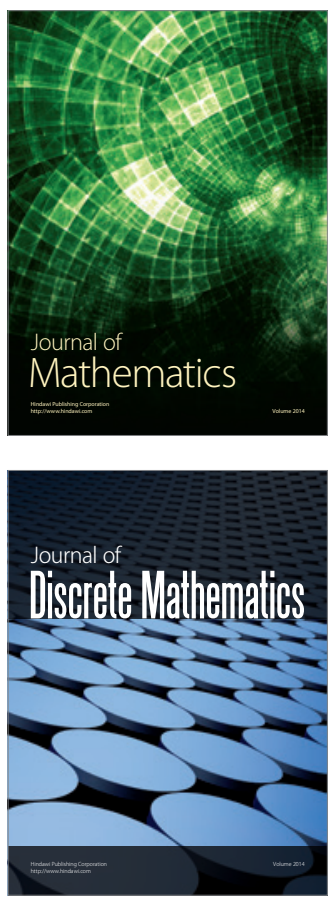

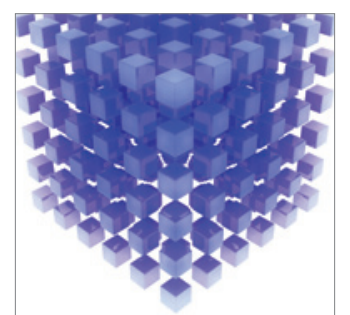

Mathematical Problems in Engineering
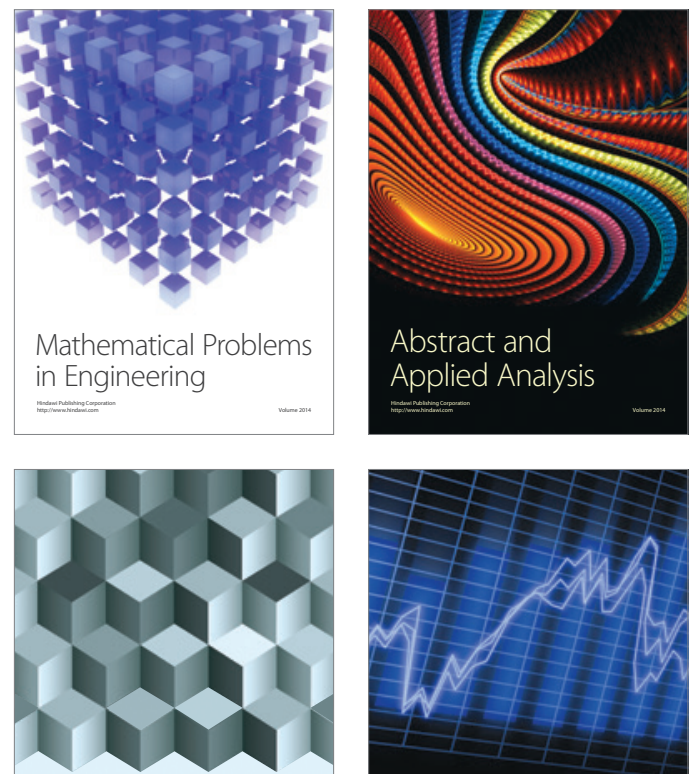

Journal of

Function Spaces

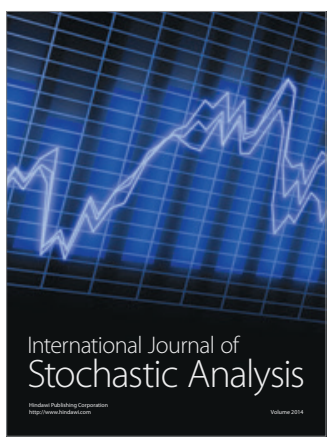

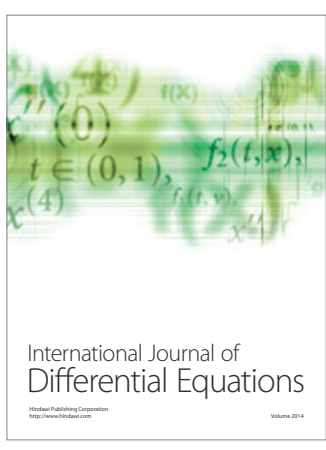
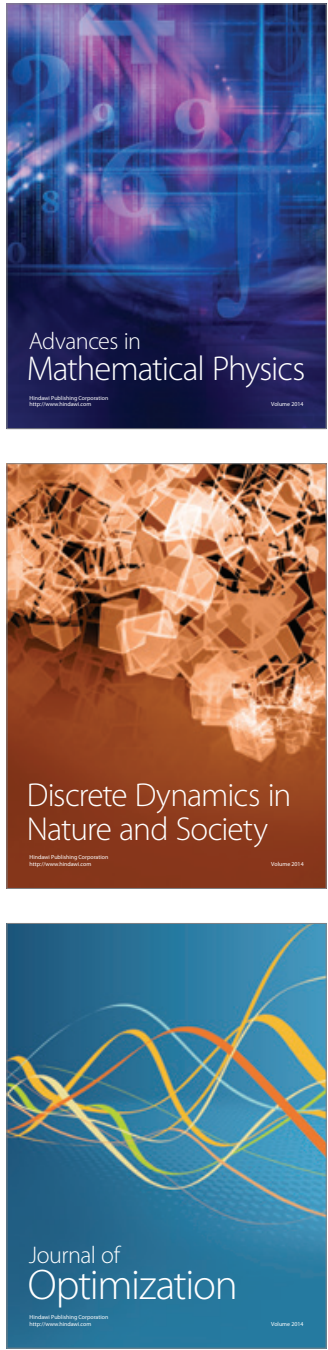